283:F663-F670.

26. Soriano, P., Montgomery, C., Geske, R., and Bradley, A. 1991. Targeted disruption of the c-Src proto-oncogene leads to osteopetrosis in mice. Cell. 64:693-702

27. Martin, G.S. 2001. The hunting of the Src. Nat. Rev Mol. Cell Biol. 2:467-475.

28. Frassetto, L., Morris, R.C., Jr., Sellmeyer, D.E., Todd, K., and Sebastian, A. 2001. Diet, evolution and aging-the pathophysiologic effects of the postagricultural inversion of the potassium-to-sodium and base-to-chloride ratios in the human diet. Eur. J. Nutr. 40:200-213.
29. Miyazaki, T., et al. 2004. Src kinase activity is essential for osteoclast function. J. Biol. Chem. 279:17660-17666.

30. Wesson, D.E., Simoni, J., and Green, D.F. 1998. Reduced extracellular $\mathrm{pH}$ increases endothelin-1 secretion by human renal microvascular endothelial cells. J. Clin. Invest. 101:578-583.

31. Khanna, A., Simoni, J., Hacker, C., Duran, M.J., and Wesson, D.E. 2004. Increased endothelin activity mediates augmented distal nephron acidification induced by dietary protein. J. Am. Soc. Nephrol. 15:2266-2275

32. Laghmani, K., Preisig, P.A., and Alpern, R.J. 2002.
The role of endothelin in proximal tubule proton secretion and the adaptation to a chronic metabolic acidosis. J. Nephrol. 15(Suppl. 5):S75-S87.

33. Orr, A.W., and Murphy-Ullrich, J.E. 2004. Regulation of endothelial cell function BY FAK and PYK2. Front. Biosci. 9:1254-1266.

34. Kodama, H., et al. 2003. Selective involvement of $\mathrm{p} 130 \mathrm{Cas} / \mathrm{Crk} / \mathrm{Pyk} 2 / \mathrm{c}-\mathrm{Src}$ in endothelin-1-induced JNK activation. Hypertension. 41:1372-1379.

35. Go, A.S., Chertow, G.M., Fan, D., McCulloch, C.E., and Hsu, C.Y. 2004. Chronic kidney disease and the risks of death, cardiovascular events, and hospitalization. N. Engl. J. Med. 351:1296-1305.

\title{
TB, or not TB: that is the question - does TLR signaling hold the answer?
}

\author{
Terence M. Doherty ${ }^{1}$ and Moshe Arditi²
}

\author{
${ }^{1}$ Atherosclerosis Research Center, Division of Cardiology, and \\ 2Department of Pediatric Infectious Diseases and Immunology, Cedars-Sinai Medical Center, Los Angeles, California, USA
}

\begin{abstract}
Innate immunity critically depends on signaling by Toll-like receptors (TLRs) that rely heavily on an intracellular adapter protein called myeloid differentiation factor 88 (MyD88). Adaptive immune defenses are generally thought to be orchestrated by innate immune responses and so should require intact TLR-MyD88 signaling pathways. But a surprising new study in MyD88-null mice infected with Mycobacterium tuberculosis challenges this view and instead suggests that MyD88 may not be absolutely required for a normal adaptive immune response (see the related article beginning on page 1790).
\end{abstract}

Every second of every day, Mycobacterium tuberculosis (MTB) infects another human being somewhere in the world (1), yet the global incidence of around 8 million cases of active tuberculosis (TB) per year is only a tiny fraction of the estimated 2 billion people infected. Only around 1 in 10 of those infected will ever develop active disease, which raises a question: why do some individuals develop disease, while others do not? The answer is not trivial: in 2002, TB caused an estimated 2 million deaths, mostly in underdeveloped countries, and over the last century, TB has killed an estimated 100 million people $(1,2)$. A solid mechanistic understanding of how MTB infection proceeds and how host defenses are marshaled against it (both successfully and unsuccessfully) has proven elusive and remains an important goal.

Nonstandard abbreviations used: IRF, IFN-regulated factor; MTB, Mycobacterium tuberculosis; MyD88, myeloid differentiation factor 88 ; NOD, nucleotide-binding oligomerization domain; TB, tuberculosis; TIR, Toll/IL-1 receptor resistance; TLR, Toll-like receptor; TRAM, TRIF-related adapter molecule; TRIF, TIR domain-containing adapter inducing IFN- $\beta$.

Conflict of interest: The authors have declared that no conflict of interest exists.

Citation for this article: J. Clin. Invest. 114:1699-1703 (2004). doi:10.1172/JCI200423867.
In this issue of the JCI, Fremond and coworkers report data that expand our understanding of the host defense against M. tuberculosis infection yet raises provocative new questions that will be sure to spark controversy (3). They used mice with a genetic deficiency in myeloid differentiation factor 88 (MyD88), a critical adapter molecule common to signaling by most Toll-like receptors (TLRs). MyD88 $/$ - mice were subject to infectious challenge with MTB and died within 4 weeks, showing evidence of massive, uncontrolled pulmonary growth of the infectious organism. Adaptive immune responses appeared to be unaffected, but iNOS activation and expression of the defensive cytokines TNF- $\alpha$, IL- 6 , and IL-12 p40 were strongly reduced in MyD88-/- macrophages and DCs. Analyses of infected lung tissues revealed massive infiltration of mononuclear cells and neutrophils in infected MyD88-null mice, as might be expected, but flow cytometric studies showed no differences in $\mathrm{CD}^{+}$and $\mathrm{CD}^{+} \mathrm{T}$ cell recruitment to lungs between infected MyD88-null and wild-type mice. These results are consistent with a normal adaptive immune response and a robust inflammatory response with increased neutrophil and macrophage recruitment in MyD88-null mice yet markedly blunted defense by innate mechanisms that eventually proved lethal. Perhaps the most intriguing finding was that vaccination of MyD88-null mice with Mycobacterium bovis BCG induced appropriate activation of $\mathrm{T}$ cells and induction of a Th1 response to mycobacterial antigens, but this only forestalled death, despite the fact that induction of cytokines (IL- $1 \beta$, IFN- $\gamma$, TNF- $\alpha$, MIP- $1 \alpha$, and MCP-1) in the lungs did not differ between MyD88-null mice and wild-type controls. The authors concluded that MyD88-dependent signaling is not significantly involved in $\mathrm{T}$ cell activation but that in the absence of MyD88, $T$ cell-mediated immunity can afford only partial protection from infection. At face value, this work would appear to offer a plausible answer to the question posed - TB, or not TB? - namely, that innate immunity in general and TLR signaling through MyD88 are essential for the host to take arms against the sea of troubles that MTB infection brings. But how does this answer square with what we know about the interaction between innate and adaptive immunity?

\section{Signaling by Toll-like receptors and MyD88 in innate immunity}

Defense against invading pathogens comes in 2 major forms. Innate immunity rapidly mounts a multipronged defense that is relatively nonspecific and relies heavily on inflammatory molecules directly or indirectly toxic to pathogens. Adaptive 


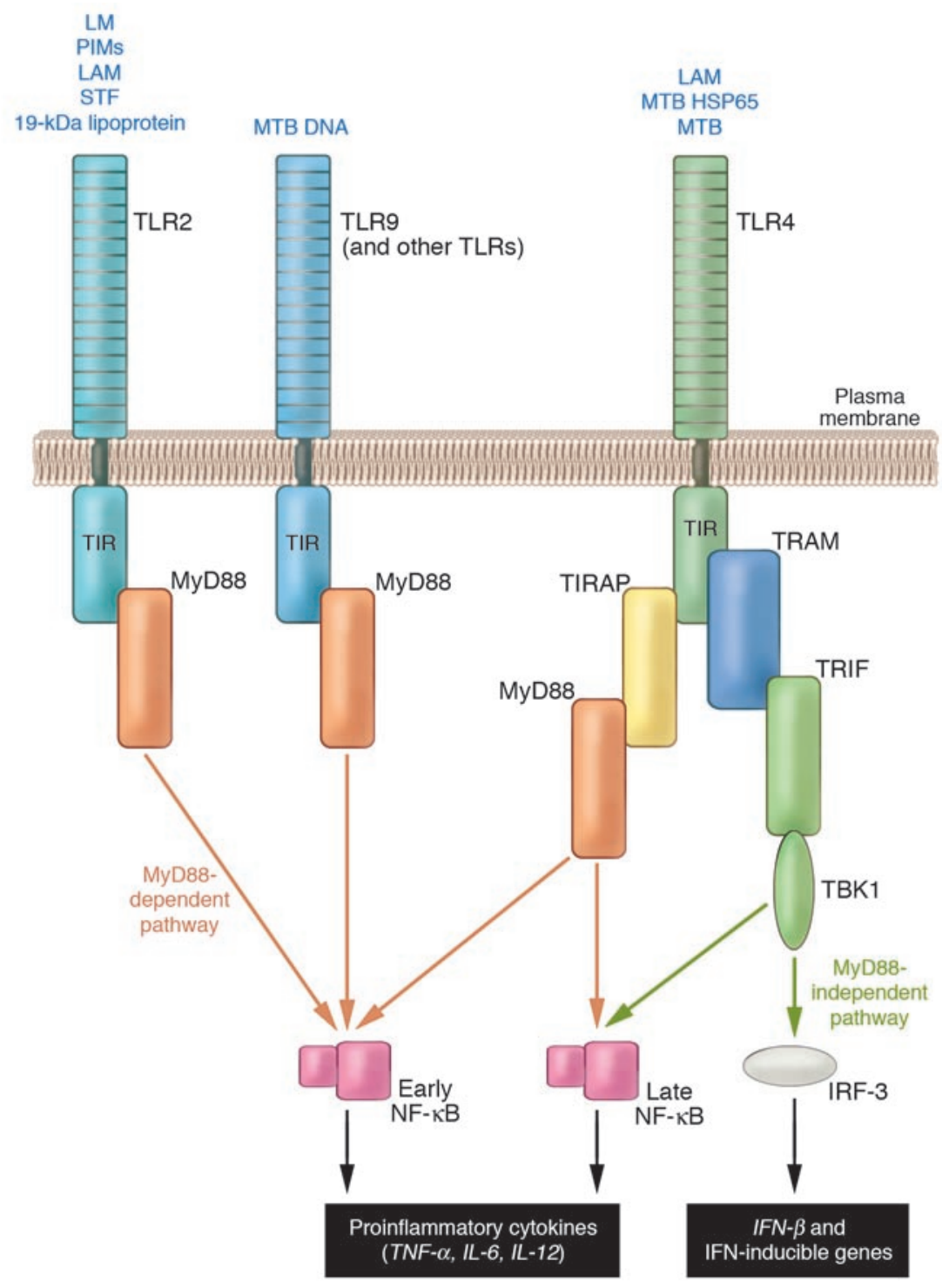

Figure 1

MyD88-dependent and MyD88-independent TLR signaling pathways. Most TLRs require MyD88; however, TLR4 can transmit signals by utilizing the alternative adapters, TRAM and TRIF. Like MyD88, this sub-pathway can activate NF- $\mathrm{B}$, but can regulate transcription of target genes in a manner that may be distinct from that elicited by the classical MyD88-dependent route. HSP65, heat shock protein 65; LAM, lipoarabinomannan; LM, lipomannan; PIMs, phosphatidyl-myoinositol mannosidases; STF, soluble tuberculosis factor; TIRAP, TIR domain-containing adapter protein.

immune defenses are deployed more slowly but involve $T$ cells and antibodies produced by leukocytes that target the pathogen with surgical precision. These 2 arms of host defense interact and cooperate in numerous ways to accomplish their task.

The discovery of the central importance of TLR-mediated signaling to innate immune mechanisms has transformed immunology and answered a fundamental question: how does the host sense the presence of foreign pathogens and how does that gen- erate a signal to rapidly mount a vigorous defense $(4,5)$ ? We now know that there are 13 mammalian TLRs that can sense molecular patterns that are common constituents of a very wide range of pathogens but are rarely found in the host. All TLRs can utilize the adapter protein MyD88 to propagate signals to gene targets and generate a rapid protective response, either by activating NF-KB or via other routes. MyD88 is also used by other inflammatory signaling pathways (e.g., IL-1 and IL-18). However, at least 2 TLRs (TLR3 and TLR4) can use alternative adapters such as Toll/IL-1 receptor resistance (TIR) domain-containing adapter inducing IFN- $\beta$ (TRIF) and TRIFrelated adapter molecule (TRAM; used by TLR4) that can activate responses distinct from those elicited by MyD88. So TLR4, for example, can utilize either the MyD88/ TIR domain-containing adapter protein or TRAM/TRIF adapter pairs to generate distinct responses (5); the latter pathway is known to be critical to the induction of DC maturation. While MyD88 typically activates NF-кB, TRAM and TRIF activate IFNregulated factor-3 (IRF-3) and IRF-7, and

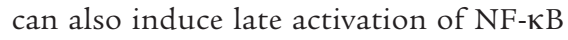
(Figure 1) (reviewed in ref. 5).

MyD88-deficient mice have an impaired ability to signal through TLRs and, consequently, their macrophages do not produce proinflammatory cytokines in response to bacterial components that are known TLR ligands (5), although TLR4 and TLR3 can link with other adapter proteins to mediate signaling in the absence of MyD88 $(5,6)$. Since TLRs directly recognize a very broad spectrum of foreign pathogens and rapidly generate a defensive response involving numerous proinflammatory cytokines, the innate immune system in MyD88-null mice is severely disabled. Besides its pivotal role in host defense, TLR signaling has also been directly implicated in an expanding list of diseases characterized by chronic inflammation, most recently atherosclerosis (7-9).

\section{How does MyD88 affect adaptive immunity?}

In 1989 Charles A. Janeway Jr. suggested that the innate immune response initiates the adaptive immune response through pattern-recognition receptors that recognize microbial products (10). Janeway's widely accepted model posits that the coupling of microbial recognition with the induction of the costimulators is accomplished by TLR signaling and is required for activation of pathogen-specific T cells (11-13). Thus, innate immunity orchestrates the adaptive response through TLR signaling. CD80 and CD86 costimulators "flag" the antigenic peptide presented by MHC molecules on the same DC as being microbe derived. Since T cells can only receive the costimulatory signal in an antigen-specific, cognate interaction with DCs, TLR-induced expression of costimulators translates the nonclonal pattern recognition signals into clonal antigen-specific immune responses (Figure 2). Thus, TLR 


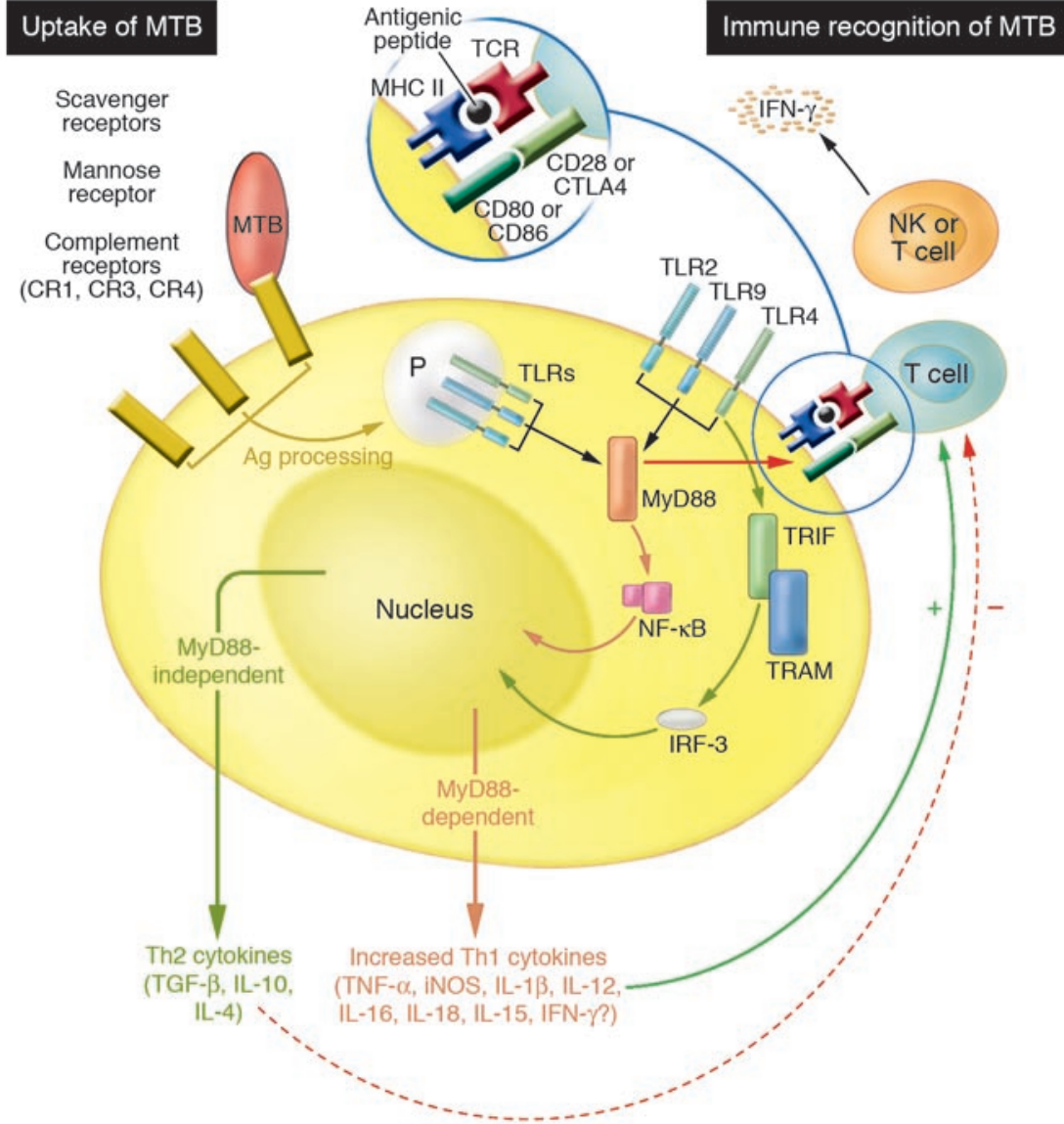

Figure 2

Bridge between innate and adaptive immunity. MTB is incorporated into phagosomes $(P)$ of APCs by one of several receptors, which appear to have a unique impact on subsequent signaling by mechanisms that are not yet clear. TLR-dependent signaling is stimulated by association of TLRs with pathogen-associated molecular patterns located on the cell surface as well as in the phagosomes. Antigenic fragments are processed and presented by $\mathrm{MHC}$ class II molecules to $T$ cells, which can then be activated provided they receive appropriate costimulatory signals transduced by engagement of T cell receptors (CD28 and CTLA-4) by CD80 (B7.1) or CD86 (B7.2) that are expressed by APCs. Normal TLR-MyD88 signaling results in rapid and potent upregulation of Th1 cytokines, which are essential for effective innate immune defense but also have a profound impact on subsequent adaptive immunity via their effects on $\mathrm{T}$ cell activation and maturation. Activated T cells and NK cells express IFN- $\gamma$, which stimulates activated APCs to continue to produce Th1 cytokines. However, in the absence of MyD88, expression of Th1 cytokines is markedly compromised, and Th2 cytokines may then predominate and inappropriately attenuate the defensive cytokine response.

signaling constitutes the essential link between innate (e.g., APCs) and adaptive (e.g., T cells) immune cells.

Once $\mathrm{CD} 4^{+} \mathrm{T}$ cells are activated, their differentiation into effector cells is controlled by a variety of factors, including cytokines produced by DCs (Figure 3). TLR-induced cytokines, such as IL-12, generally specialize in the induction of Th1 responses. Mice deficient in MyD88, in contrast, are severely compromised in Th1 differentiation and instead generate mostly Th2 responses $(11,14)$. These data are consistent with the view that TLRs primarily control the induction of Th1 but not Th2 responses (13).

Immune responses are tailored to the threat posed by specific types of microbes (reviewed in ref. 15). For example, intracellular pathogens and mycobacterial infections instigate a robust Th1 response for clearance, but helminthic infestation generates a Th2 response. Therefore, it is likely that distinct combinations of activated TLRs triggered by each microbe might dictate the outcome that occurs, and TLRs are perhaps best thought of as a kind of microbial "barcode reader" (16). Exactly how the complete response to the barcode occurs is not clear, but it is likely that phagosome and cell surface signaling are differentially involved (Figure 2).

But in MyD88-deficient mice, TLR signaling is severely disabled. How then, in the study by Fremond et al. (3), could adaptive responses to $\mathrm{MTB}$-specific antigens be preserved? This is difficult to answer right now. On one hand, we do not yet know enough about the specific TLR signaling events that mediate the response to MTB infection. That said, it seems likely that MyD88 deficiency critically interferes with one or more steps in the process $(17,18)$. For example, phagocytosis of the pathogen can be accomplished by a number of different receptors present on professional APCs; the particular receptor involved appears to be an important determinant of intracellular fate and the subsequent immune response. Some TLR signaling originates from phagosomes and might be expected to control specific aspects of the overall response. Separately, recognition of MTB at the cell surface by TLRs is also an important determinant of the effectiveness of the host response. MTB products can be recognized by TLR 2 or TLR4, and in addition, TLR9 can sense the presence of MTB DNA (Figure 1). Production of both inflammatory and anti-inflammatory cytokines and chemokines is another key step in the defensive response that may be particularly relevant to the findings of Fremond et al. (3). Molecules that generate TLR2-dependent signals are entirely dependent upon MyD88, but as noted above, TLR4 has MyD88-independent options available for signaling. It is possible, then, that some of these processes could be mediated by TLR4 in a MyD88independent manner. Alternatively, other innate immune receptors such as nucleotide-binding oligomerization domain (NOD) may play a more important role than presently understood. Obviously, considerable work needs to be done to clarify these issues.

\section{Immune mechanisms in mycobacterial infection: controversies}

The work of Fremond et al. (3) generates interesting questions and adds to a growing controversy. First of all, is it really true that MyD88 is not involved in regulating the adaptive immune response to MTB? 


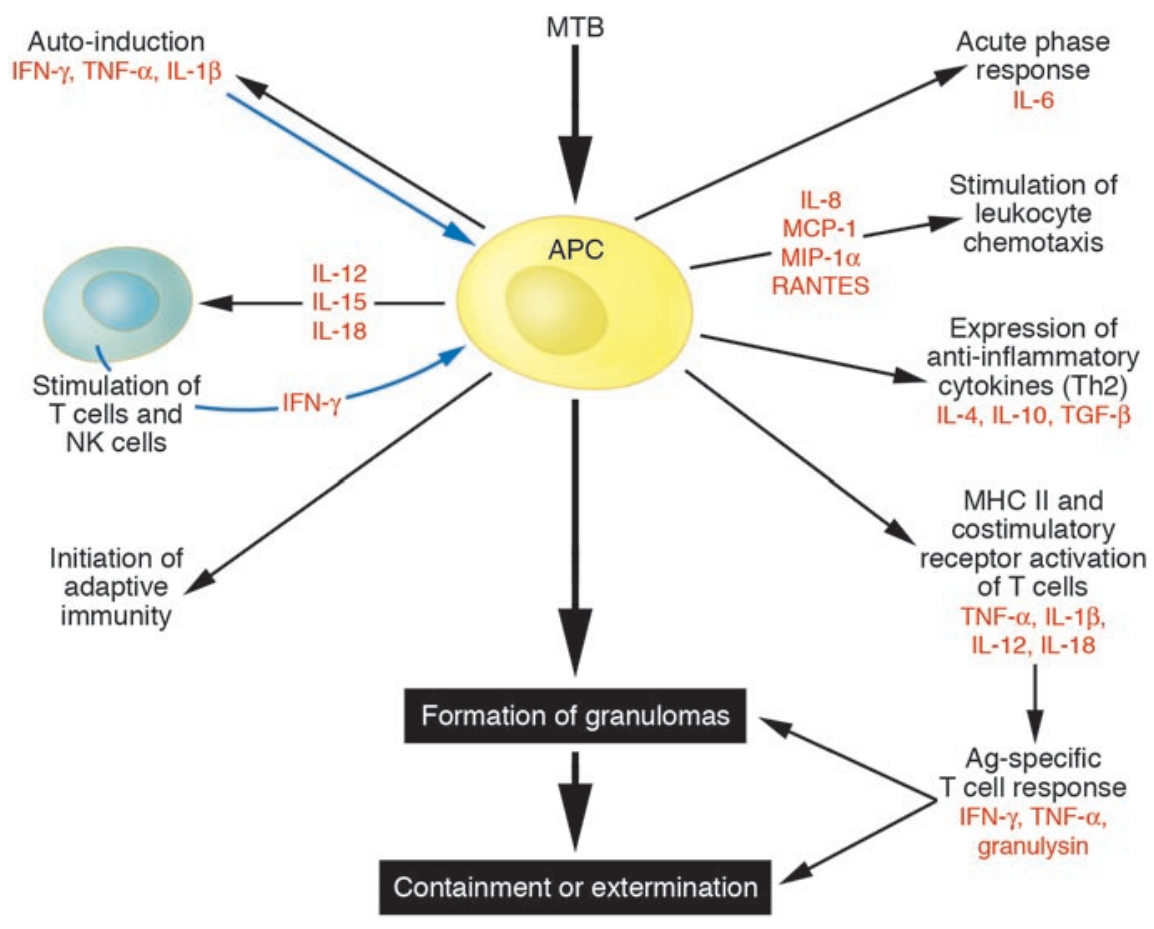

Figure 3

Multifaceted immune responses to MTB. Professional APCs such as macrophages and DCs mount a multifaceted response to pathogen invasion that most often contains or eliminates MTB, but MyD88-dependent innate immune mechanisms appear to be critical to accomplish this task.

Scanga et al. (19) first established that host resistance to infection with MTB is dependent on MyD88 and therefore strongly implicated TLR signaling in this response, since mice deficient in the IL-1 or IL-18 signaling pathway show only minor alterations in the normal defense to MTB infection (reviewed in ref. 19). There is evidence to corroborate this and suggest that the principle may be more general. MyD88-null mice have been shown to be highly susceptible to a number of pathogens, including Listeria monocytogenes (20), Staphylococcus aureus (21), Toxoplasma gondii (22), and Mycobacterium avium (23). In the study by Scanga et al. (19), expression of IL-12, TNF- $\alpha$, IFN- $\gamma$, and nitric oxide synthase 2 were markedly decreased but not completely eliminated in MTB-infected MyD88-deficient mice, which indicates a severe impairment in the normal adaptive immune response to this infection. In another study, adaptive immunity and antigen-specific $T$ cell activation were significantly impaired in $\mathrm{CD}^{+} \mathrm{T}$ cells from MyD88-/- mice infected with $M$. avium (23). Therefore, it is unclear why adaptive immunity would be impaired for $M$. avium infection but conserved for M. tuberculosis, as reported by Fremond et al. (3).
Surprisingly, mice deficient in TLR2 (24, 25), TLR4 $(24,26,27)$, or TLR6 (25), all of which rely heavily (but not exclusively in the case of TLR4) on MyD88, show no defects in acute resistance to aerogenic MTB infection. Another study reported that MyD88-null mice may not always succumb to MTB infection (28). Furthermore, a recent study determined that gene expression in macrophages infected with MTB in vitro is largely MyD88 independent (29). On the other hand, mice deficient in MyD88 could mount an apparently normal adaptive response against the intracellular pathogen Listeria monocytogenes (30) and Borrelia burgdorferi (31), which suggests (like Fremond et al.; ref. 3) that MyD88 may not always be needed for an effective adaptive defense. Other data are consistent with this conclusion as well: mice deficient in both MyD88 and TRIF have no TIR domain signaling yet have normal lymphoid organs and lymphoid architecture and normal immunoglobulin levels, which suggests that alternative pathways must exist for adaptive immune activation (Bruce Beutler, personal communication). These findings clearly underscore the substantial complexity in host defenses, whereby essential or redundant roles for specific components are dependent both on the nature of the microbe and perhaps also the site of infection.

Reconciling these discordant results is not straightforward. Possible explanations could include differences in MTB strains used, differences in the nature and the dose of the microbe, and differences in genetic backgrounds of MyD88-null mice. But it can be tentatively suggested that MyD88 regulates resistance to at least some strains of MTB. But if this is true, how is it accomplished? Does MyD88 control genetic programs only within the jurisdiction of innate immunity, or does it have a more general function in modulating crucial interactions between innate and adaptive immunity? In this regard, Fremond's conclusion that there is no significant impact of MyD88 on the adaptive response is at odds with the generally accepted model that adaptive immunity is controlled by innate mechanisms and therefore, by extension, MyD88 (13). That said, the contribution of other innate signaling mechanisms besides TLRs to regulation of the adaptive response to MTB infection has not been fully defined. For example, the intracellular NOD proteins, which recognize pathogen-associated molecular patterns independently of TLR signaling, can activate NF- $\mathrm{KB}$ and increase production of IFN- $\gamma$ (32). In addition, double-stranded RNA can elicit an innate response independently of TLR3 and TRIF (33).

\section{TB or not TB:}

\section{the unanswered questions}

For now, these data and the current study by Fremond et al. (3) should make one thing very clear: we do not yet understand the signaling mechanisms, transcriptional responses, subcellular processes, or cellcell interactions that follow MTB infection. This leads us to a series of provocative questions directly relevant to the work of Fremond et al. Is adaptive immunity really dependent upon innate immunity? If MyD88 is not involved in regulating the adaptive response to MTB infection, then what is? What are the MTB-derived ligands and the TLRs that instigate signaling? TLR signaling involves complex and variable assemblies of cofactors and adapters for efficient transduction; how are expression and availability of these accessory molecules regulated, and how important might that be to the response? Does interaction among TLRs or downstream crosstalk play a key role in the response? What are the 
functions of cell surface signaling by TLRs in MTB infection compared with signaling that originates from intracellular sources such as phagosomes and endosomes? Are there different genetic programs controlled by these? How is the entire machinery fine tuned to prevent an inappropriate degree of inflammation, and how is it turned off when it is no longer necessary?

An intriguing model that may at least partly resolve some of the discrepancies outlined above suggests that the TLRdependent response of APCs is defined by the cell type and the niche in which the cell resides. Closely related lineages that express similar repertoires of TLRs may nevertheless respond very differently to the presence of TLR ligands (13). And finally, McCaffrey and colleagues recently reported that distinct gene clusters may be induced by particular pathogens depending on their subcellular localization in a manner that is independent of TLRs and MyD88 (34).

\section{What dreams may come?}

The questions posed outline fertile ground for future investigations that will doubtless provide insights that we, like Hamlet, can only wonder about. And the same dilemma that beset Hamlet-"To be, or not to be: that is the question" - eons earlier also confronted evolution: how best to respond to the slings and arrows of pathogenic invasion by MTB? Be it coincidence or sublime irony, the resolution arrived at by evolution is the same as Hamlet's:

But that the dread of something after death,
The undiscover'd country from whose bourn
No traveller returns, puzzles the will...
Thus conscience does make cowards of us all.

- William Shakespeare, Hamlet, act 3, scene 1

The internal drives of Hamlet and evolution continue to fuel efforts to take arms against a sea of troubles, to understand how immune defenses protect us from MTB, and so to develop more effective ways to counter the ability of pathogens to cause us to shuffle off this mortal coil. The work of Fremond et al. (3) now takes us a step further along this path.

\section{Acknowledgments}

The authors are supported by grants from the NIH (HL 66436 and AI 058128, to M. Arditi).

Address correspondence to: Moshe Arditi, Cedars-Sinai Medical Center, Department of Pediatric Infectious Diseases and Immunology, 8700 Beverly Boulevard, Los Angeles, California 90048, USA. Phone: (310) 423-4471; Fax: (310) 423-8284; E-mail: moshe.arditi@cshs.org.

1. World Health Organization. 2004. Tuberculosis. Fact sheet number 104. http://who.int/mediacentre/factsheets/fs104/en/.

2. Frieden, T.R., Sterling, T.R., Munsiff, S.S., Watt, C.J., and Dye, C. 2003. Tuberculosis. Lancet. 362:887-899.

3. Fremond, C.M., et al. 2004. Fatal Mycobacterium tuberculosis infection despite adaptive immune response in the absence of MyD88. J. Clin. Invest. 114:1790-1799. doi:10.1172/JCI200421027.

4. Beutler, B. 2004. Inferences, questions and possibilities in Toll-like receptor signalling. Nature. 430:257-263.

5. Akira, S., and Takeda, K. 2004. Toll-like receptor signalling. Nat. Rev. Immunol. 4:499-511.

6. Palsson-McDermott, E.M, and O'Neill, L.A.J. 2004 Signal transduction by the lipopolysaccharide receptor, Toll-like receptor-4. Immunology. 113:153-162.

7. Michelsen, K.S., et al. 2004. Lack of Toll-like receptor 4 or myeloid differentiation factor 88 reduces atherosclerosis and alters plaque phenotype in mice deficient in apolipoprotein E. Proc. Natl. Acad. Sci.U. S. A. 101:10679-10684.

8. Bjorkbacka, H., et al. 2004. Reduced atherosclerosis in MyD88-null mice links elevated serum cholesterol levels to activation of innate immunity signaling pathways. Nat. Med. 10:416-421.

9. Michelsen, K.S., Doherty, T.M., Shah, P.K., and Arditi, M. 2004. TLR signaling: an emerging bridge from innate immunity to atherogenesis. J. Immunol. 173:5901-5907.

10. Janeway, C.A., Jr. 1989. Approaching the asymptote? Evolution and revolution in immunology. Cold Spring Harb. Symp. Quant. Biol. 54:1-13.

11. Schnare M., et al. 2001. Toll-like receptors control activation of adaptive immune responses Nat. Immunol. 2:947-950.

12. Janeway, C.A., Jr., and Medzhitov, R. 1998. Introduction: the role of innate immunity in the adaptive immune response. Semin. Immunol. 10:349-350.

13. Iwasaki, A., and Medzhitov, R. 2004. Toll-like receptor control of the adaptive immune responses. Nat. Immunol. 5:987-995.

14. Kaisho, T., et al. 2002. Endotoxin can induce MyD88-deficient dendritic cells to support Th2 cell differentiation. Int. Immunol. 14:695-700.

15. Hoebe, K., Janssen, E., and Beutler, B. 2004. The interface between innate and adaptive immunity. Nat. Immunol. 5:971-974.

16. Aderem, A. 2003. Phagocytosis and the inflammatory response. J. Infect. Dis. 187:S340-S345.

17. van Crevel, R., Ottenhoff, T.H., and van der Meer, J.W. 2002. Innate immunity to Mycobacterium tuberculosis. Clin. Microbiol. Rev. 15:294-309.
18. Quesniaux, V., et al. 2004. Toll-like receptor pathways in the immune responses to mycobacteria. Microbes Infect. 6:946-959.

19. Scanga, C.A., et al. 2004. MyD88-deficient mice display a profound loss in resistance to Mycobacterium tuberculosis associated with partially impaired Th1 cytokine and nitric oxide synthase 2 expression. Infect. Immun. 72:2400-2404.

20. Seki, E., et al. 2002. Critical role of myeloid differentiation factor 88-dependent proinflammatory cytokine release in early phase clearance of Listeria monocytogenes in mice. J. Immunol. 169:3863-3868.

21. Takeuchi, O., Hoshino, K., and Akira, S. 2000. Cutting edge: TLR2-deficient and MyD88-deficient mice are highly susceptible to Staphylococcus aureus infection. J. Immunol. 165:5392-5396.

22. Scanga, C.A., et al. 2002. Cutting edge: MyD88 is required for resistance to Toxoplasma gondii infection and regulates parasite-induced IL-12 production by dendritic cells. J. Immunol. 168:5997-6001.

23. Feng, C.G., et al. 2003. Mice lacking myeloid differentiation factor 88 display profound defects in host resistance and immune responses to Mycobacterium avium infection not exhibited by Toll-receptor 2 (TLR2)- and TLR4-deficient animals. J. Immunol. 171:4758-4764.

24. Reiling, N., et al. 2002. Cutting edge: Toll-like receptor (TLR2)- and TLR4-mediated pathogen recognition in resistance to airborne infection with Mycobacterium tuberculosis. J. Immunol. 169:3480-3484.

25. Sugawara, I., et al. 2003. Mycobacterial infection in TLR2 and TLR6 knockout mice. Microbiol. Immunol. 47:327-336.

26. Abel, B., et al. 2002. Toll-like receptor 4 expression is required to control chronic Mycobacterium tuberculosis infection in mice. J. Immunol. 169:3155-3162.

27. Shim, T.S., Turner, O.C., and Orme, I.M. 2003. Toll-like receptor 4 plays no role in susceptibility of mice to Mycobacterium tuberculosis. Tuberculosis (Edinb.). 83:367-371.

28. Sugawara, I., Yamada, H., Mizuno, S., Takeda, K., and Akira, S. 2003. Mycobacterial infection in MyD88-deficient mice. Microbiol. Immunol. 47:841-847.

29. Shi, S., et al. 2003. MyD88 primes macrophages for full-scale activation by interferon-gamma yet mediates few responses to Mycobacterium tuberculosis. J. Exp. Med. 198:987-997.

30. Way, S.S., Kollmann, T.R., Hajjar, A.M., and Wilson, C.B. 2003. Protective cell-mediated immunity to Listeria monocytogenes in the absence of myeloid differentiation factor 88. J. Immunol. 171:533-537

31. Liu, N., Montgomery, R.R., Barthold, S.W., and Bockenstedt, L.K. 2004. Myeloid differentiation antigen 88 deficiency impairs pathogen clearance but does not alter inflammation in Borrelia burgdorferi-infected mice. Infect. Immun. 72:3195-3203.

32. Philpott, D.J., and Girardin, S.E. 2004. The role of Toll-like receptors and Nod proteins in bacterial infection. Mol. Immunol. 41:1099-1108.

33. Hoebe, K., et al. 2003. Upregulation of costimulatory molecules induced by lipopolysaccharide and double-stranded RNA occurs by Trif-dependent and Trif-independent pathways. Nat. Immunol. 4:1223-1229.

34. McCaffrey, R.L., et al. 2004. A specific gene expression program triggered by Gram-positive bacteria in the cytosol. Proc. Natl. Acad. Sci. U. S. A. 101:11386-11391. 